\title{
Targeted and non-targeted liquid chromatography-mass spectrometric workflows for identification of transformation products of emerging pollutants in the aquatic environment
}

\author{
Anna A. Bletsou a , Junho Jeon ${ }^{\mathrm{b}, \mathrm{c}}$, Juliane Hollender ${ }^{\mathrm{b}, \mathrm{d}}$, Eleni Archontaki ${ }^{\mathrm{a}}$, \\ Nikolaos S. Thomaidis a,* \\ a Laboratory of Analytical Chemistry, Department of Chemistry, University of Athens, Panepistimiopolis Zographou, 15771 Athens, Greece \\ ${ }^{\mathrm{b}}$ Eawag, Swiss Federal Institute of Aquatic Science and Technology, 8600 Dübendorf, Switzerland \\ c Department of Environmental Engineering, Changwon National University, Changwon, 641-773 Gyeongnam, Republic of Korea \\ d Institute of Biogeochemistry and Pollutant Dynamics (IBP), ETH Zurich, 8092 Zurich, Switzerland
}

\section{A R T I C L E I N F O}

\section{Keywords:}

Abiotic degradation

Aquatic environment

Biodegradation

Emerging pollutant

Liquid chromatography

Mass spectrometry

Non-target screening

Suspect screening

Target screening

Transformation product

\begin{abstract}
A B S T R A C T
Identification of transformation products (TPs) of emerging pollutants is challenging, due to the vast number of compounds, mostly unknown, the complexity of the matrices and their often low concentrations, requiring highly selective, highly sensitive techniques. We compile background information on biotic and abiotic formation of TPs and analytical developments over the past five years. We present a database of biotic or abiotic TPs compiled from those identified in recent years. We discuss mass spectrometric (MS) techniques and workflows for target, suspect and non-target screening of TPs with emphasis on liquid chromatography coupled to MS (LC-MS). Both low- and high-resolution (HR) mass analyzers have been applied, but HR-MS is the technique of choice, due to its high confirmatory capabilities, derived from the high resolving power and the mass accuracy in MS and MS/MS modes, and the sophisticated software developed.
\end{abstract}

(c) 2014 Elsevier B.V. All rights reserved.

\section{Contents}

1. Introduction

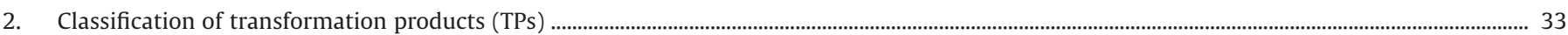

2.1. Transformation products formed by biotic reactions ................................................................................................................................................. 34

2.1.1. Transformation products formed by microbial metabolism ............................................................................................................................ 34

2.1.2. Transformation products (metabolites) formed by human metabolism ................................................................................................... 34

2.1.3. Transformation products formed in wildlife .......................................................................................................................... 34

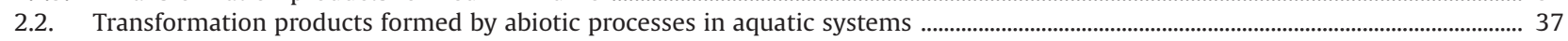

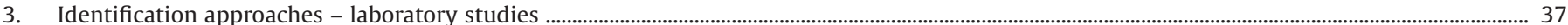

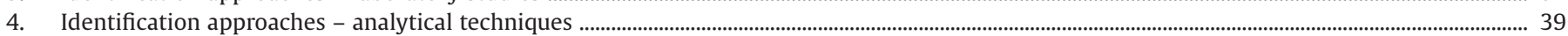

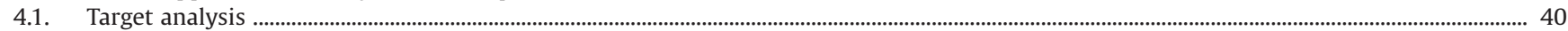

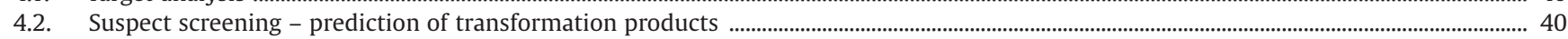

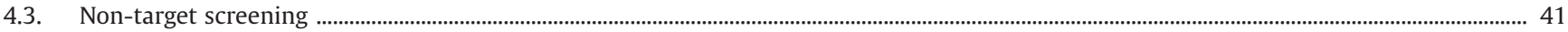

5. Future needs and trends

Acknowledgments .............................................................................................................................................................................................................

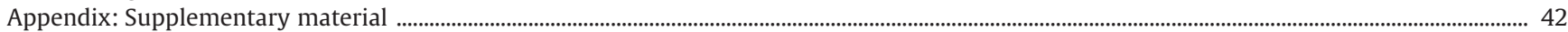

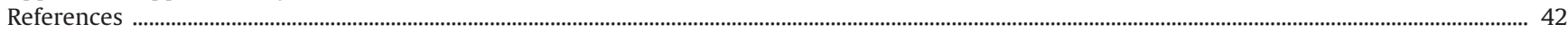

\footnotetext{
* Corresponding author. Tel.: +30210 7274317; Fax: +30 2107274750.

E-mail address: ntho@chem.uoa.gr (N.S. Thomaidis).
}

\section{Introduction}

The term "emerging pollutants" (EPs) [or “emerging contaminants" (ECs)] refers to compounds and their metabolites that are not currently covered by existing water-quality regulations, have not been studied often, are overlooked and are thought to be 
potential threats to environmental ecosystems and human health and safety. According to NORMAN (Network of reference laboratories, research centers and related organizations for monitoring emerging environmental substances), they are compounds that are not included in routine environmental monitoring programs and may be candidates for future legislation due to their adverse effects and/or persistency (http://www.norman-network.net/). Most regulating and implementation bodies, responsible for water and wastewater treatment, are working on the assumption that the socalled priority pollutants are responsible for the most significant share of environmental, human health and economic risk, even though they represent a minor fraction of the universe of known and yet-to be identified chemicals [1].

EPs encompass a diverse group of compounds, including pharmaceuticals and personal-care products (PPCPs), drugs of abuse (DoAs) and their metabolites, steroids and hormones, endocrinedisrupting compounds, surfactants, perfluorinated compounds, phosphoric ester flame retardants, industrial additives and agents (e.g., benzotriazoles and benzothiazoles), siloxanes, artificial sweeteners, and gasoline additives.

Once released into the environment, EPs are subject to biotic and abiotic transformation processes that are responsible for their transformation and/or elimination, according to their persistence, transport, and ultimate destination. Various transformations can take place, producing compounds that, to some extent, differ in their environmental behavior and ecotoxicological profile from the parent compound. Formation of transformation products (TPs) occurs mainly through oxidation, hydroxylation, hydrolysis, conjugation, cleavage, dealkylation, methylation and demethylation. EPs and their TPs can move vertically through the soil profile to groundwater and away from the source site with mobile groundwater. They also have the potential to reach surface water when they travel laterally as surface run-off or through sub-soil tile drains, entering streams, major rivers, reservoirs, and ultimately estuaries and oceans [2].

Since there is a gap in the information on the occurrence and the toxicity of TPs in the environment, we are unable to evaluate their significance in risk assessment $[3,4]$. Standardized toxicity tests can provide quantitative information on the toxicity of the TP, compared to its parent compound, but these studies are limited [5-7]. In general, TPs are less toxic and more polar than the parent compounds. However, in some cases, they may be more persistent or exhibit higher toxicity or be present at much higher concentrations [8].

Although there is legislation regulating chemicals [e.g., pesticides, veterinary drugs, and persistent organic pollutants (POPs)], there is little mention of their TPs. Concerns over the TPs of pesticides in plants have been expressed since 1991 (European Directive 91/414/EEC), while the term "metabolite" appears in Regulation (EC) $1107 / 2009$, concerning plant-protection products, and in Directives 2001/82/EC and 98/8/EC, concerning veterinary medical and biocidal products, respectively. European Medicines Agency (EMEA, 2006) also referred to the need for assessment of potential environmental risks of human medicinal products. However, in all these documents, there is no clarification on the determination, limits and toxicological effects of metabolites or TPs.

In OECD guidelines, concerning the Aerobic and Anaerobic Transformation in Aquatic Sediment Systems, adopted in 2002, it is claimed that TPs detected at $\geq 10 \%$ of the applied radioactivity should be identified. Meanwhile, EU Regulation 1907/2006 (REACH) requires identification of major TPs and degradation products for the registration of the substance. In the Regulation (EC) 850/2004 on POPs, a reference to their transformation processes also exists.

There is therefore a clear need to reveal the qualitative and quantitative occurrence of TPs in the environment, but this is only possible with continual development of instrumental analysis. Thereby, the range of identifiable chemicals is extended, and the quantification limits are lowered. With respect to obtaining a holistic view of risk, target-based environmental monitoring should be accompanied by non-target analysis using high-resolution (HR) hybrid mass spectrometers. The development of these highly resolved, accurate, hybrid, tandem mass spectrometers, and improved sophisticated software, has enabled more reliable, selective target analysis of highly polar compounds, and screening for unknown pollutants. The major benefit of full-scan and HR, accurate MS is that, within a single analytical run, target, suspect and non-target compounds can be analyzed or identified.

In the analysis of EPs, HR mass spectrometry (HR-MS) has been widely reported [3,9-12]. Moreover, for identification of TPs in environmental, food and biological samples, hybrid HR mass analyzers [e.g., linear ion trap Orbitrap MS and quadrupole time-of-flight MS (Q-TOF)] have been used, following specific workflows [12-16]. More specifically, human and microbial metabolites, oxidation and photodegradation TPs of pharmaceuticals have been discussed often [17-21]. Similarly, TPs of pesticides in biological (human metabolism, phase II), food and environmental samples have been reviewed $[22,23]$. Furthermore, the TPs of anthelmintics [24]. UV filters in the environment $[25,26]$ and steroidal compounds in biological samples [27] are included in recent review papers. An interesting fact concerning the analysis of EPs and their TPs is enantioselective biotransformation. Chiral EPs or chiral TPs formed may have enantioselective activity or toxicity, making chiral chromatography indispensable [28].

Achievements, future trends and new developments in the analysis of EPs and their TPs were summarized by Farré et al. [29] and Fisher et al. [30]. Recently, highly sophisticated, comprehensive, stepwise workflows were also presented by Moschet et al. [31] and Hug et al. [32] for suspect and non-target screening of pesticides and EPs, including TPs in their suspect lists. However, it is still challenging to profile TPs in environment samples, since they are formed through many possible reactions, automatic workflows for the identification are not readily available, so manual data inspection is necessary, though time consuming, and, finally, there are no standards available.

The aim of this review is to compile the recent information regarding the background of (biotic and abiotic) transformation of EPs. We provide a brief overview of existing literature on transformation studies under biotic and abiotic conditions in recent years and we compile a list of all the EPs studied and comprehensive information for researchers in the field. We briefly summarize target analysis, since the development of accurate mass instruments and sophisticated computer tools has led to suspect and non-target analysis, even though all three procedures are indispensable parts of an integrated approach to determination of EPs and their TPs. We present the design of laboratory studies to facilitate identification of TPs by LC-MS and appropriate sample preparation. We thoroughly discuss target, suspect and non-target workflows using HRMS/MS to identify new TPs.

\section{Classification of transformation products (TPs)}

TPs occurring in the environment can be classified into two main categories: biotransformation products formed by biotic or abiotic processes. This classification has subcategories that we describe in detail below, emphasizing the aquatic environment. The biotransformation products include human, animal and microbial metabolites in engineered and natural systems. The abiotic TPs are the outcome of hydrolysis, photolytic and photocatalytic degradation in the natural environment and water-treatment processes (e.g., chlorination, ozonation and advanced oxidation). 


\subsection{Transformation products formed by biotic reactions}

TPs are formed by microbial activities in natural and engineered environmental compartments, such as soil, surface water or wastewater treatment. Enzymatic reactions are involved in microbial transformation [such as, oxidation (e.g., hydroxylation, N- and S-oxidation, and dealkylation) and reduction (e.g., dehalogenation, nitro reduction, and hydrolysis of amides and carboxyl esters)]. As mentioned, pharmaceuticals, drugs of abuse and other chemicals consumed by humans and other mammals can be metabolized and then released into the environment as metabolites. For mammals, besides oxidation and reduction, conjugation reactions also occur with endogenous molecules, such as carbohydrates, sulfate, glutathione and amino acids.

Some environmental pollutants are significantly accumulated and subsequently transformed in wildlife. In particular, aquatic organisms are considered the primary receptors, and might show qualitatively and quantitatively different metabolic pathways compared to microbes and humans. Hence, the metabolites formed in aquatic organisms and their toxicity are of increasing ecotoxicological concern.

In the sections below, we discuss biotransformation products, classified into three groups, and we present findings from recent studies, with a clearly stated identification workflow in Table 1. Table S1 (Supplementary Material) shows an extensive list of all the biotransformation products of EPs presented so far in the literature.

\subsubsection{Transformation products formed by microbial metabolism}

Due to the increasing occurrence of EPs in wastewaters, the formation of TPs during biological treatment and the mechanisms involved have been investigated for various classes of compounds \{i.e., antibiotics [35,45,54], analgesics (painkillers) [39], anticonvulsants [37], anti-inflammatories [40,41], iodinated X-ray contrast media (ICM) $[46,47]$ and anti-viral/-bacterial/-fungal agents $[33,56,57]\}$. Most of the studies were performed in batch systems with activated sludge.

In almost all the studies, oxidative reactions, such as hydroxylation, oxidation, and dealkylation, were observed as the primary biotransformation mechanisms. Hydroxylated metabolites were identified for triclosan [57], codeine [39], diclofenac [40,41], sulfapyridine [54], and a UV filter [48]. In some cases, oxidation of hydroxyl groups was followed by oxidative decarboxylation, deacetylation, and dealkylation taking place at the amide moieties [33,46,47]. Molecules with $\mathrm{N}-, \mathrm{O}-$, or S-alkyl groups were probably transformed to dealkylated forms, as observed for naproxen [53], triclosan [57], diclofenac [41] and ICM [46,47]. Other oxidative reactions included ring opening, oxidative deamination and oxidative dechlorination $[34,45,50,56]$.

Helbling et al. intensively investigated microbial transformation of 30 xenobiotic compounds with amide groups and observed 53 TPs resulting from amide hydrolysis, N-dealkylation, hydroxylation, oxidation, dehalogenation, glutathione conjugation and many more pathways [34]. Moreover, the hydrolysis rate and the dominant reaction related to the degree of alkyl substitution of the amide group.

Other studies investigated predictive factors of the biotransformation reactions. Ammonia removal and ammonia monooxygenase (amoA)-transcript abundance can be associated with oxidative micropollutant-biotransformation reactions, without necessarily being catalyzed by amoA [58].

Meanwhile, reduction reactions predominantly take place under anaerobic conditions, such as reductive dechlorination of 5-chlorobenzotriazole and chlorpromazine and dehydration and hydration of testosterone [38,55]. However, nitro reduction of N,Ndiethyl 1-4-nitrobenzamide was evident under aerobic conditions in a recent study [34].
Apart from common oxidation and reduction, other reactions, such as decarboxylation and deacetylation, were reported less frequently $[46,47]$. In addition, conjugation reactions, such as phosphorylation, succinylation and glutathione substitution, are also possible biotransformation mechanisms [34,45].

The biodegradability of perfluorinated compounds (PFCs) attracts the interest of scientists. A number of studies presented the degradability and the fate of PFC precursors, such as fluorotelomer alcohols in sediments resulting in perfluorinated carboxylic acids $[43,44]$. Nevertheless, no TPs of perfluoroctanoate (PFOA) have been reported, stressing that these compounds are very stable and hardly degradable $[59,60]$.

\subsubsection{Transformation products (metabolites) formed by human metabolism}

Even though human metabolites of PPCPs and their mechanisms of formation have been extensively studied in pharmacology, limited information on their occurrence and stability in the environment is available. However, human and microbial metabolisms partly present the same metabolic reactions and thus the same metabolites, so their discrimination in environmental samples is sometimes difficult. Kern et al. stated that six pharmaceutical TPs found in surface-water samples were known human metabolites of metamizole, aminopyrine, carbamazepine and verapamil from registration files [51]. However, four of these six metabolites were formed through epoxidation, dihydroxylation and O-demethylation, which can also take place in microbial metabolism. Perez and Barceló reported that hydroxylation products of diclofenac and aceclofenac, known as both human and microbial metabolites, were measured in wastewater samples [41].

Mass balances of influent and effluent samples can clarify the origin of the TPs in more detail. In the tiered approach proposed by Kern et al., batch experiments with activated sludge can be used to verify the findings and to quantify transformation rates [52].

For estrogenic compounds, the metabolites formed are glucuronide and sulfate conjugates and are frequently detected in untreated wastewaters [61]. However, the conjugated estrogens are vulnerable in aerobic activated sludge and end up as free estrogens after de-conjugation [62]. The de-conjugation behavior is also observed for pharmaceuticals, resulting in negative removal efficiencies $[63,64]$. However, deconjugation reactions happen with different reaction rates. For example, for estrogens, sulfate conjugates are reported to be more persistent than glucuronides. In case of lamotrigine, N-glucuronide metabolite has frequently been detected in wastewater, surface-water and groundwater samples, unlike $\mathrm{O}$-glucuronide, due to the difference in degradability of $\mathrm{O}$ - and $\mathrm{N}$-glucuronide products [65].

\subsubsection{Transformation products formed in wildife}

EPs are ubiquitous in the environment due to their low degradability, and probably accumulate in biota (bioaccumulation) and sediments. Biotransformation in organisms, as a subsequent process of bioaccumulation, is of great interest in order to clarify the fate and the toxicity of those compounds and their TPs. However, studies of drug metabolism in fish are extremely limited and the metabolic pathway and enzymes responsible for the metabolism of the drugs in fish are largely unknown [66].

Moreover, metabolites of POPs have been measured in various tissues (e.g., blood, blubber, fat, and bird eggs) and in marine organisms (including fish), but, in many cases, it is not known if the compounds formed are the result of in-vivo metabolism or are bioaccumulated from the environment [67].

In a study, oxidation reactions in vitro were hindered by the increased number of bromine substituents and hydroxylated metabolites and oxidative bond-cleavage products are formed in fish 
Table 1

Identified biotransformation products of emerging contaminants in the aquatic environment

\begin{tabular}{|c|c|c|c|c|c|c|c|}
\hline \multirow{2}{*}{$\begin{array}{l}\text { Parent compound/Group of } \\
\text { substances }\end{array}$} & \multirow{2}{*}{$\begin{array}{l}\text { (Bio)assay/Test organism/ } \\
\text { Degradation system }\end{array}$} & \multirow{2}{*}{$\begin{array}{l}\text { Identified TPs/Transformation } \\
\text { reaction }\end{array}$} & \multirow{2}{*}{$\begin{array}{l}\text { Toxicity } \\
\text { Information }\end{array}$} & \multicolumn{3}{|c|}{ Identification workflow } & \multirow[t]{2}{*}{ Ref. } \\
\hline & & & & $\begin{array}{l}\text { Sample } \\
\text { preparation }\end{array}$ & $\begin{array}{l}\text { Instrumental } \\
\text { technique(s) }\end{array}$ & $\begin{array}{l}\text { Software (prediction/ } \\
\text { post data treatment) }\end{array}$ & \\
\hline Acyclovir (ACV), Penciclovir (PCV) & $\begin{array}{l}\text { Batch systems seeded with } \\
\text { activated sludge }\end{array}$ & $\begin{array}{l}\text { ACV: carboxy-ACV, PCV: } 8 \text { TPs } \\
\text { (oxidation) }\end{array}$ & - & SPE (Isolute ENV+) & $\begin{array}{l}\text { HPLC-LTQ-Orbitrap-MS", } \\
\text { 1D \& 2D NMR }\end{array}$ & $\begin{array}{l}\text { UM-PPS, Thermo XCalibur, } \\
\text { fragmentation pattern }\end{array}$ & [33] \\
\hline 30 Amide-containing compounds & $\begin{array}{l}\text { Batch system seeded with sludge } \\
\text { from a pilot-scale membrane } \\
\text { bioreactor }\end{array}$ & $\begin{array}{l}53 \text { TPs: amide hydrolysis and } \\
\text { N-dealkylation, hydroxylation, } \\
\text { oxidation, ester hydrolysis, } \\
\text { dehalogenation, nitro reduction, and } \\
\text { glutathione conjugation/kinetic study }\end{array}$ & - & - & $\begin{array}{l}\text { LC-MS Orbitrap, data } \\
\text { dependent MS/MS } \\
\text { acquisition }\end{array}$ & $\begin{array}{l}\text { UM-PPS, Thermo XCalibur, } \\
\text { post-acquisition data } \\
\text { processing of the full-scan } \\
\text { MS data (mass accuracy, } \\
\text { isotope matching and MS/ } \\
\text { MS evaluation) }\end{array}$ & [34] \\
\hline Amoxicillin & $\begin{array}{l}\text { Laboratory alkaline } \& \text { acidic } \\
\text { hydrolysis study with } \\
\text { wastewater and river water }\end{array}$ & 4 TPs: b-lactam ring cleavage & - & SPE (OASIS $\left.{ }^{\text {TMHLB}}\right)$ & LC/QTOF-MS/MS & $\begin{array}{l}\text { Agilent MassHunter } \\
\text { Workstation, mass } \\
\text { accuracy, XIC, } \\
\text { fragmentation pattern }\end{array}$ & [35] \\
\hline $\begin{array}{l}\text { Benzotriazoles: 1H-BTR } \\
\text { 5- } \mathrm{CH}_{3}-\mathrm{BTR} \\
\text { 4-CH }-\mathrm{CH}_{3} \text {-BTR }\end{array}$ & $\begin{array}{l}\text { Batch studies under aerobic } \\
\text { conditions with sludge }\end{array}$ & $\begin{array}{l}42 \text { candidate TPs - } 4 \text { confirmed TPs: } \\
1-\mathrm{CH}_{3}-\mathrm{BTR}, 4-\mathrm{OH}-\mathrm{BTR}, 5-\mathrm{OH}-\mathrm{BTR} \text { (from } \\
1 \mathrm{H}-\mathrm{BTR} \text { ) and 5-COOH-BTR (from 5-CH }{ }_{3-}^{-} \\
\text {BTR). Hydroxylation at the benzene ring } \\
\text { was the major pathway }\end{array}$ & - & - & $\begin{array}{l}\text { LC-MS Orbitrap, data } \\
\text { dependent MS/MS } \\
\text { acquisition }\end{array}$ & $\begin{array}{l}\text { Suspect screening after } \\
\text { UM-PPS, non-target } \\
\text { screening; Thermo } \\
\text { XCalibur, post-acquisition } \\
\text { data processing of the full- } \\
\text { scan MS data; In silico } \\
\text { fragmentation by MetFrag; } \\
\text { MOLGEN-MS/MS for } \\
\text { structure annotation }\end{array}$ & [36] \\
\hline Carbamazepine & $\begin{array}{l}\text { Aqueous medium from air } \\
\text { pulsed fluidized bioreactor and } \\
\text { from culture broth }\end{array}$ & $\begin{array}{l}\text { Acridone, acridine, 10,11-dihydro-10,11- } \\
\text { epoxycarbamazepine \& dihydroxy- } \\
\text { carbamazepine }\end{array}$ & $\begin{array}{l}\text { Acute toxicity } \\
\text { test: Vibrio } \\
\text { fischeri } \\
\text { luminescence } \\
\text { reduction: } \\
\text { nontoxic }\end{array}$ & SPE (OASIS $\left.{ }^{\mathrm{TM} H L B}\right)$ & $\begin{array}{l}\text { UPLC/ESI-QqToF - } \\
\text { HPLC/ESI-QqLIT - }\end{array}$ & $\begin{array}{l}\text { Waters MassLynx, XIC, } \\
\text { mass accuracy, } \\
\text { fragmentation pattern }\end{array}$ & [37] \\
\hline $\begin{array}{l}\text { Chlorpromazine (tricyclic } \\
\text { antipsychotic drug) }\end{array}$ & $\begin{array}{l}\text { OECD: Closed Bottle test, } \\
\text { Manometric } \\
\text { Respirometry test, modified } \\
\text { Zahn-Wellens test, anaerobic } \\
\text { test }\end{array}$ & $\begin{array}{l}\text { TPs: } 3 \text { aerobic } \& 1 \text { anaerobic } \\
\text { (hydroxylation, demethylation, } \\
\text { acetylation) }\end{array}$ & $\begin{array}{l}\text { neither readily } \\
\text { nor inherently } \\
\text { biodegradable } \\
\text { - toxic to } \\
\text { anaerobic } \\
\text { sludge }\end{array}$ & - & $\begin{array}{l}\text { HPLC-UV-Fluorescence- } \\
\text { IT MS }^{n}\end{array}$ & $\begin{array}{l}\text { fragmentation \& isotopic } \\
\text { pattern, Bruker Esquire } \\
\text { software, Metabolite } \\
\text { Detect (Metabolite Tools), } \\
\text { Data Analysis }\end{array}$ & [38] \\
\hline Codeine (Opium Alkaloid) & $\begin{array}{l}\text { Aerobic batch experiments } \\
\text { seeded with activated sludge }\end{array}$ & $\begin{array}{l}8 \text { TPs: double bond shifts, introduction } \\
\text { of hydroxy groups, amine demethylation } \\
\text { - transformation pathways suggested } \\
\text { for structurally related opium alkaloids } \\
\text { (morphine \& dihydrocodeine) }\end{array}$ & - & SPE (OASIS $\left.{ }^{\mathrm{TM} M C X}\right)$ & $\begin{array}{l}\text { HPLC-LTQ-Orbitrap-MS } \\
\text { \& HPLC-Qq-LIT-MS, data } \\
\text { dependent acquisition, } \\
\text { 1D \& 2DNMR }\end{array}$ & $\begin{array}{l}\text { fragmentation pattern, } \\
\text { Thermo Xcalibur }\end{array}$ & [39] \\
\hline Diclofenac & $\begin{array}{l}\text { fungus Trametes versicolor - in } \\
\text { vivo and in vitro experiments } \\
\text { with cytochrome P450 and } \\
\text { laccase }\end{array}$ & $\begin{array}{l}\text { 4-hydroxydiclofenac, } \\
\text { 5-hydroxydiclofenac (hydroxylation) \& } \\
\text { 4-(2,6-dichlorophenylamino)-1,3- } \\
\text { benzenedimethanol }\end{array}$ & $\begin{array}{l}\text { Decrease in } \\
\text { ecotoxicity } \\
\text { (Microtox test) }\end{array}$ & - & HPLC-UV, NMR & - & [40] \\
\hline Diclofenac, Aceclofenac & pilot MBR & $\begin{array}{l}3 \text { novel TPs: nitrosation, nitration, } \\
\text { N-dealkylation and carboxylation }\end{array}$ & - & $\begin{array}{l}\text { SPE (Oasis }{ }^{\mathrm{TM}} \mathrm{HLB}, \\
\text { Isolute ENV+) }\end{array}$ & $\begin{array}{l}\text { HPLC/ESI-QqLIT-MS, } \\
\text { UPLC/ESI-QqTOF-MS, } \\
\text { H/D-Exchange } \\
\text { Experiments }\end{array}$ & $\begin{array}{l}\text { fragmentation pattern, } \\
\text { Waters MassLynx }\end{array}$ & [41] \\
\hline $\begin{array}{l}\text { Flame retardants: 4,4- } \\
\text { dibromodiphenyl ether (BDE 15), } \\
\text { 2,2,4,4-tetrabromo-diphenyl } \\
\text { ether (BDE 47), tetrabromo- } \\
\text { bisphenol A (TBBPA) }\end{array}$ & $\begin{array}{l}\text { in vitro - liver microsomes and } \\
\text { S9 fractions of crucian carp }\end{array}$ & $\begin{array}{l}\text { BDE 15: Bromophenol, } \\
\text { 2monohydroxylateddibromodiphenyl } \\
\text { ethers } \\
\text { TBBPA: 2,6-dibromo-4-isopropyl-phenol }\end{array}$ & - & LLE & $\begin{array}{l}\text { HPLC-QqQ-MS/MS, GC- } \\
\text { IT-MS/MS }\end{array}$ & $\begin{array}{l}\text { fragmentation pattern, } \\
\text { Thermo XCalibur }\end{array}$ & [42] \\
\hline Fluorotelomer alcohols (6:2 FTOH) & Batch study with river sediment & $\begin{array}{l}\text { 5:3 acid, PFPeA, PFHxA, and PFBA, 5:2 } \\
\text { sFTOH }\end{array}$ & - & $\begin{array}{l}\text { ENVI }^{\mathrm{TM}} \text {-Carb } \\
\text { (graphitized carbon) }\end{array}$ & $\begin{array}{l}\text { LC-MS/MS (QqQ) -SRM } \\
\text { technique }\end{array}$ & - & [43] \\
\hline
\end{tabular}




\begin{tabular}{|c|c|c|c|c|c|c|c|}
\hline \multirow{2}{*}{$\begin{array}{l}\text { Parent compound/Group of } \\
\text { substances }\end{array}$} & \multirow{2}{*}{$\begin{array}{l}\text { (Bio)assay/Test organism/ } \\
\text { Degradation system }\end{array}$} & \multirow{2}{*}{$\begin{array}{l}\text { Identified TPs/Transformation } \\
\text { reaction }\end{array}$} & \multirow{2}{*}{$\begin{array}{l}\text { Toxicity } \\
\text { Information }\end{array}$} & \multicolumn{3}{|c|}{ Identification workflow } & \multirow[t]{2}{*}{ Ref. } \\
\hline & & & & $\begin{array}{c}\text { Sample } \\
\text { preparation }\end{array}$ & $\begin{array}{l}\text { Instrumental } \\
\text { technique(s) }\end{array}$ & $\begin{array}{l}\text { Software (prediction/post } \\
\text { data treatment) }\end{array}$ & \\
\hline $\begin{array}{l}\text { 5:3 Polyfluorinated acid (major TP } \\
\text { of 6:2 FTOH \& 8:2 FTOH) }\end{array}$ & Activated sludge & $\begin{array}{l}4: 3 \text { acid, } 3: 3 \text { acid, one-carbon removal } \\
\left.\text { pathways(- }-\mathrm{CF}_{2}-\right) \text {, perfluoropentanoic } \\
\text { acid (PFPeA), perfluorobutanoic acid } \\
(\mathrm{PFBA})\end{array}$ & - & $\begin{array}{l}\text { C18 cartridge, ENVI }{ }^{\mathrm{TM}}- \\
\text { Carb }\end{array}$ & $\begin{array}{l}\text { HPLC-LTQ Orbitrap MS/ } \\
\text { MS }\end{array}$ & $\begin{array}{l}\text { Mass defect data filtration, } \\
\text { mass accuracy }\end{array}$ & {$[44]$} \\
\hline $\begin{array}{l}\text { Fluoroquinolones (norfloxacin, } \\
\text { ciprofloxacin) Macrolides } \\
\text { (azithromycin, erythromycin, } \\
\text { roxitromycin) }\end{array}$ & $\begin{array}{l}\text { Wastewater effluents from } \\
\text { Zenon hollow-fiber membrane } \\
\text { bioreactor (MBR) }\end{array}$ & $\begin{array}{l}\text { fluoroquinolones: conjugate compounds } \\
\text { formed by phosphorylation, (phase II } \\
\text { metabolites) macrolides: conjugates } \\
\text { formed by succinylation of the } \\
\text { piperazine ring \& smaller metabolites } \\
\text { formed by oxidative break-up of } \\
\text { piperazine moiety }\end{array}$ & - & SPE (OASIS HLB) & $\begin{array}{l}\text { UHPLC-QTOFMS, MS/MS } \\
\text { acquisition }\end{array}$ & Waters MassLynx & [45] \\
\hline $\begin{array}{l}\text { Iodinated X-ray Contrast Media } \\
\text { (iohexol, iomeprol, iopamidol) }\end{array}$ & $\begin{array}{l}\text { Batch reactor seeded with } \\
\text { secondary wastewater effluents }\end{array}$ & $\begin{array}{l}27 \text { TPs: oxidation, oxidative } \\
\text { decarboxylation, deacetylation, cleavage } \\
\text { at the amide moieties }\end{array}$ & - & SPE (Isolute ENV+) & $\begin{array}{l}\text { HPLC-Qq-LIT-MS, MS/MS, } \\
\text { NMR }\end{array}$ & fragmentation pattern & [46] \\
\hline $\begin{array}{l}\text { Iodinated X-ray Contrast Media } \\
\text { (diatrizoate, iohexol, iomeprol, } \\
\text { iopamidol) }\end{array}$ & $\begin{array}{l}\text { Aerobic soil-water and river } \\
\text { sediment-water batch systems }\end{array}$ & $\begin{array}{l}7 \text { novel TPs: Oxidation, cleavage of } \mathrm{N}-\mathrm{C} \\
\text { bonds and decarboxylation }\end{array}$ & - & - & HPLC-Qq-LIT-MS, MS/MS & fragmentation pattern & [47] \\
\hline $\begin{array}{l}4^{\prime} \text {-methylbenzylidene- camphor } \\
\text { (4-MBC) }\end{array}$ & Fungus Trametes versicolor & $\begin{array}{l}\text { Hydroxylated and pentose-conjugated } \\
\text { TPs }\end{array}$ & $\begin{array}{l}\text { No endocrine } \\
\text { disruption and } \\
\text { dioxin-like } \\
\text { activity }\end{array}$ & $\begin{array}{l}\text { Pressurized liquid } \\
\text { extraction (PLE) }\end{array}$ & UPLC-QqTOF-MS/MS & $\begin{array}{l}\text { fragmentation pattern, } \\
\text { Waters MassLynx }\end{array}$ & [48] \\
\hline Metformin & $\begin{array}{l}\text { Closed Bottle test, Manometric } \\
\text { Respiratory test, Zahn-Wellens } \\
\text { test with activated sludge }\end{array}$ & $\begin{array}{l}\text { Guanyl urea (dealkylation \& oxidative } \\
\text { deamination) }\end{array}$ & - & - & HPLC-Ion Trap MS & Bruker Esquire & [49] \\
\hline $\begin{array}{l}6 \text { Pharmaceuticals \& } 6 \text { Pesticides } \\
\text { (atenolol, bezafibrate, diazepam, } \\
\text { levetiracetam, oseltamivir, } \\
\text { valsartan \& carbetamide, } \\
\text { clomazone, DEET, napropamide, } \\
\text { propachlor, tebutam) }\end{array}$ & $\begin{array}{l}\text { Batch reactors seeded with } \\
\text { activated sludge }\end{array}$ & $\begin{array}{l}21 \text { TPs from suspect screening \& } 26 \text { TPs } \\
\text { from non-targeted screening }\end{array}$ & - & - & $\begin{array}{l}\text { HPLC-LTQ-Orbitrap, data } \\
\text { dependent MS/MS } \\
\text { acquisition }\end{array}$ & $\begin{array}{l}\text { UM-PPS (suspects), } \\
\text { Thermo XCalibur, detailed } \\
\text { post-acquisition data } \\
\text { processing of the full-scan } \\
\text { MS data (target screening, } \\
\text { non-target screening) }\end{array}$ & [50] \\
\hline $\begin{array}{l}52 \text { Pesticides, Biocides, } \\
\text { Pharmaceuticals }\end{array}$ & Surface water & 19 plausible TPs identified & - & $\begin{array}{l}\text { SPE (100 mg Strata-X- } \\
\text { AW, } 100 \mathrm{mg} \\
\text { Strata-X-CW, } 150 \mathrm{mg} \\
\text { Isolute ENV+, } 200 \mathrm{mg} \\
\text { Oasis HLB) }\end{array}$ & $\begin{array}{l}\text { HPLC-LTQ-Orbitrap MS/ } \\
\text { MS }\end{array}$ & $\begin{array}{l}\text { UM-PPS, Thermo Xcalibur, } \\
\text { Mass Frontier, mass } \\
\text { accuracy, fragmentation } \\
\text { pattern, isotopic pattern }\end{array}$ & [51] \\
\hline $\begin{array}{l}\text { Pharmaceuticals: atenolol, } \\
\text { bezafibrate, ketoprofen, } \\
\text { metoprolol, ranitidine, valsartan, } \\
\text { venlafaxine, carbendazim }\end{array}$ & Sludge-seeded batch reactors & $12 \mathrm{TPs}$ & - & $\begin{array}{l}\text { SPE (Strata-X-AW, } \\
\text { Strata-X-CW, Isolute } \\
\text { ENV+, Oasis HLB) }\end{array}$ & $\begin{array}{l}\text { HPLC-LTQ-Orbitrap, data } \\
\text { dependent MS/MS } \\
\text { acquisition }\end{array}$ & $\begin{array}{l}\text { UM-PPS, Thermo Xcalibur, } \\
\text { detailed workflow for the } \\
\text { identification of } \\
\text { biotransformation } \\
\text { products }\end{array}$ & [52] \\
\hline $\begin{array}{l}\text { Acidic pharmaceuticals } \\
\text { (ketoprofen, bezafibrate, } \\
\text { naproxen, ibuprofen, diclofenac) }\end{array}$ & $\begin{array}{l}\text { Activated sludge as inocculum } \\
\text { under aerobic conditions }\end{array}$ & $\begin{array}{l}\text { Ketoprofen: } 2 \text { novel TPs Bezafibrate: } \\
\text { 4-chlorobenzoicacid Naproxen: } \\
\text { O-desmethyl-naproxen Ibuprofen: } 2 \\
\text { isomers of hydroxy-ibuprofen }\end{array}$ & - & $\begin{array}{l}\text { ion-pair solid phase } \\
\text { extraction (IP-SPE) }\end{array}$ & $\begin{array}{l}\text { LC-ESI-MS/MS (QqQ), } \\
\text { Full scan MS, Product ion } \\
\text { scan, LC-UV }\end{array}$ & - & [53] \\
\hline Sulfapyridine Sulfathiazole & $\begin{array}{l}\text { Fluidized bed reactor with } \\
\text { Trametes versicolor seeded with } \\
\text { sterilized sewage sludge, in vitro } \\
\text { and in vivo assays with purified } \\
\text { laccase }\end{array}$ & 7 TPs formylated and hydroxylated & - & - & UPLC-QqTOF-MS & $\begin{array}{l}\text { mass accuracy, } \\
\text { fragmentation pattern }\end{array}$ & [54] \\
\hline Testosterone & $\begin{array}{l}\text { Gamma proteobacterium } \\
\text { Steroidobacter denitrificans }\end{array}$ & $\begin{array}{l}10 \text { TPs (dehydrogenation, } \\
\text { hydrogenation) }\end{array}$ & - & - & $\begin{array}{l}\text { HPLC-APCI-LTQ-Orbitrap, } \\
\text { MS/MS, LC-UV, LC - ion } \\
\text { trap - MS, NMR }\end{array}$ & $\begin{array}{l}\text { mass accuracy, } \\
\text { fragmentation pattern }\end{array}$ & [55] \\
\hline Triclosan & laccase in aqueous systems & $\begin{array}{l}\text { 2,4-dichlorophenol (2,4-DCP), } \\
\text { dechlorinated forms of 2,4-DCP, } \\
\text { 2-chlorohydroquinone }\end{array}$ & $\begin{array}{l}\text { Detoxication by } \\
\text { enzymatic } \\
\text { transformation }\end{array}$ & - & $\begin{array}{l}\text { GC-IT-MS, HPLC-ESI- } \\
\text { QqQ, MS/MS, LC-UV }\end{array}$ & $\mathrm{XIC}$ & [56] \\
\hline
\end{tabular}


liver from 4,4'-dibromodiphenyl ether and tetrabromobisphenol A, but not from higher brominated flame retardants [42].

Recently, metabolites of polar organic pollutants formed in freshwater crustaceans, used in risk assessment, were identified $[68,69]$. Jeon et al. found 25 metabolites of irgarol, terbutryn, tramadol and venlafaxine in Gammarus pulex and Daphnia magna, formed via oxidation reactions including $\mathrm{N}$ - and $\mathrm{O}$-demethylation, hydroxylation, $\mathrm{N}$-oxidation and glutathione conjugation [68]. This shows the relevance of metabolism in wildlife.

\subsection{Transformation products formed by abiotic processes in aquatic systems}

Abiotic TPs are formed by water-treatment processes and in the aquatic environment by hydrolysis and photolysis. Table 2 shows research papers studying the formation of TPs from EPs under abiotic conditions through one of the above processes, from the past three years, with a clearly described identification workflow. Table S2 (Supplementary Material) shows a more extensive list of studies. Also, review papers provide information for TPs of antibiotics and estrogens already identified, covering a wide range of abiotic processes [76,77].

Oxidation processes, such as chlorination, chloramination, ozonation, and advanced oxidation by $\mathrm{UV} / \mathrm{H}_{2} \mathrm{O}_{2}$ treatment, are the major processes used in advanced water treatment for disinfection and removal of ECs [8]. The oxidative reaction mechanisms often rely on the formation of reactive, short-lived oxygen-containing intermediates, such as hydroxyl radicals $(\bullet \mathrm{OH})$. Generally, the TPs formed correlate with the process conditions [e.g., the physicochemical properties of the matrix, and the specific conditions of treatment (e.g., time and medium)].

Ozone is a strong oxidant that can be used as a more selective agent for the removal of micropollutants. Ozonation may take place by the direct reaction of the ozone molecule with the target compound or by means of hydroxyl radicals produced from the decomposition of ozone in aqueous media. In practice, both direct and indirect reactions take place simultaneously. Ozone was recently used as a fourth full-scale treatment step in wastewater treatment [78]. Next to ozonation TPs, by-products formed by oxidation of matrix components, such as carcinogenic $\mathrm{N}$-nitrosodimethylamine (NDMA) and bromate, have to be taken into the cost-benefit analysis of such technology [78].

Chlorination is a chemical process commonly used in water treatment for disinfection. In most cases, chlorination is not applied when oxidation of organic micropollutants is the goal, because it can produce biologically active TPs [79]. Especially when the inorganic content in the water matrix is very high, some reactive species, such as chloride or sulfate radicals, are produced, and they directly influence the formation of TPs. The chlorine radical $(\mathrm{Cl} \bullet$ ) may lead to the formation of chlorinated organic compounds, which are known to be very harmful, and, in some cases, able to generate persistent substances [80]. In this disinfection process, hypochlorous acid ( $\mathrm{HClO})$ is the reagent mainly responsible for pathogen destruction, but both $\mathrm{HClO}$ and $\mathrm{ClO}^{-}$react with organic compounds, giving addition, substitution, or oxidation products. One of the major concerns regarding disinfection byproducts from chlorination is that $\mathrm{NaClO}$ is known to produce genotoxic TPs and can thus increase the acute toxicity [75].

Photochemistry represents an important degradation process in the environment or as a light-related, advanced treatment of water. Many studies have been carried out on direct and indirect photolytic or photocatalytic degradation of EPs. For pesticides, mineralization of pesticides by photocatalytic degradation has been reported and the by-products and intermediates of organophosphate pesticides by photocatalytic degradation were recently presented [81]. Pharmaceutical compounds [19,82], endocrinedisrupting compounds [82], UV filters [83], and phenol [84] have also been thoroughly surveyed for their fate and their TPs during photolysis.

\section{Identification approaches - laboratory studies}

Simulation of the transformation processes in batch experiments under well-defined conditions with appropriate controls is a very common first approach for the identification of TPs. Batch experiments can be applied under biotic and abiotic conditions at high concentrations of the parent EPs.

For biodegradation experiments, samples can be provided directly from a wastewater-treatment plant (WWTP) or a pilotscale WWTP (ps-WWTP) or from natural waters [33,34,51]. Moreover, the ability of microorganisms to degrade EPs has been studied in Erlenmeyer flasks with various microorganisms under study, such as fungus Trametes versicolor and bacterial Pseudomonas strains [37,40]. Parameters, such as $\mathrm{pH}$, dissolved oxygen $\left(\mathrm{O}_{2}\right)$, temperature (T) and total suspended solids (TSS), have to be monitored and adjusted to allow direct comparison with environmental conditions. In the case of batch reactors seeded with activated sludge or wastewater, a concentration of $3 \mathrm{~g} / \mathrm{L}$ of total suspended solids (TSS) is desirable in order to avoid matrix interferences. During such experiments, spiked and non-spiked samples run in parallel, as do spiked autoclaved diluted sludge or autoclaved groundwater and ultrapure water in order to correct for abiotic processes [39]. Samples are collected periodically so that the reaction kinetics of the analytes can be determined sufficiently [33,50].

Whereas the question may arise if the use of batch experiments can simulate the biotransformation of EPs in full-scale WWTPs, previous studies with batch reactors have proved that, indeed, biotransformation reactions and kinetics can be observed reasonably well in a batch reactor [58]. In aerobic conditions, reactors are loosely capped and shaken or mixed in order to allow free transfer of oxygen, while, for anaerobic treatment, the process is conducted under an atmosphere of $\mathrm{N}_{2}$. To maintain anaerobic conditions, the anaerobic chamber is flushed with a mixture of $\mathrm{N}_{2}$ / $\mathrm{CO}_{2}$ gas.

Membrane bioreactors (MBRs), air-pulsed fluidized-bed bioreactors and sequencing batch reactors (SBRs) [37,50] are more realistic systems than batch reactors, because they better simulate the conditions in the full-scale system.

To assess the principal biodegradability and the formation of potential TPs, tests of the OECD series are referred to in the literature:

- the widely used Closed Bottle test (CBT, OECD 301 D) working with low bacterial density;

- the manometric respirometry test (MRT, OECD $301 \mathrm{~F}$ ) working with medium bacterial density; and,

- the Zahn-Wellens test (ZWT, OECD 302 B) working with high bacterial density.

The CBT simulates conditions in surface water, while the other two simulate effluents [38]. However, those OECD tests usually work at very high concentrations and provide no reaction kinetics.

When preliminary studies in batch experiments are completed, verification of the results should then be carried out using real environmental samples. However, as concentrations are usually lower in the environmental systems, analytical methods have to be adapted. There are few studies with direct injection of environmental samples for the identification of TPs. More often, sediments, activated sludge, wastewater and river-water samples were collected and subjected to solid-phase extraction (SPE) or liquidliquid extraction (LLE) prior to analysis, for enrichment of the analytes $[35,44,51]$. To achieve sufficient enrichment for a broad range of compounds, including TPs with different physical-chemical properties, Kern et al. used simultaneously four different SPE sorbents in one 
Table 2

Identified transformation products (TPs) of emerging contaminants formed by abiotic processes (e.g., ozonation, photolysis and chlorination) in water

\begin{tabular}{|c|c|c|c|c|c|c|c|}
\hline \multirow{2}{*}{$\begin{array}{l}\text { Parent compound/Group of } \\
\text { substances }\end{array}$} & \multirow{2}{*}{$\begin{array}{l}\text { Type of study/ } \\
\text { Degradation } \\
\text { process }\end{array}$} & \multirow[t]{2}{*}{ Identified TPs } & \multirow{2}{*}{$\begin{array}{l}\text { Toxicity } \\
\text { information }\end{array}$} & \multicolumn{3}{|c|}{ Identification workflow } & \multirow[t]{2}{*}{ Ref. } \\
\hline & & & & $\begin{array}{c}\text { Sample } \\
\text { preparation }\end{array}$ & $\begin{array}{l}\text { Instrumental } \\
\text { technique(s) }\end{array}$ & $\begin{array}{c}\text { Software } \\
\text { (prediction/post } \\
\text { data treatment) }\end{array}$ & \\
\hline Azithromycin & $\begin{array}{l}\text { Photodegradation: } \\
\text { Simulated solar } \\
\text { radiation }\end{array}$ & 7 TPs - pathway suggested & - & - & $\begin{array}{l}\text { UPLC/ESI-QqToF-MS, UPLC/ESI- } \\
\text { QqQ-MS }\end{array}$ & $\begin{array}{l}\text { Waters MassLynx, mass accuracy, } \\
\text { fragmentation pattern, double-bond } \\
\text { equivalents }\end{array}$ & [70] \\
\hline Benzotriazoles & Ozonation & $\begin{array}{l}11 \text { oxidation TPs (with and } \\
\text { without ring cleavage) }\end{array}$ & - & SPE (Isolute ENV+) & $\begin{array}{l}\text { HPLC-QTOF, UV-Vis, full scan mode } \\
\text { HPLC-QqQ-MS/MS, HPTLC/AMD } \\
\text { H/D Exchange, Derivatization } \\
\text { experiments }\end{array}$ & $\begin{array}{l}\text { XIC, Agilent MassHunter Workstation, } \\
\text { Molecular Formula Generator, } \\
\text { Molecular Feature Extractor, Mass } \\
\text { Profiler Professional }\end{array}$ & [71] \\
\hline $\begin{array}{l}\text { Chlorpromazine (tricyclic } \\
\text { antipsychotic drug) }\end{array}$ & $\begin{array}{l}\text { Photodegradation: } \\
\text { UV/VIS xenon lamp }\end{array}$ & 28 TPs & - & - & $\begin{array}{l}\text { HPLC-UV-Fluorescence-Ion Trap- } \\
\text { MS }^{\mathrm{n}}\end{array}$ & $\begin{array}{l}\text { Esquire software, Bruker Metabolite } \\
\text { Tools \& Data Analysis, fragmentation \& } \\
\text { isotopic pattern }\end{array}$ & [38] \\
\hline Cocaine, benzoylecgonine (BE) & $\begin{array}{l}\text { Hydrolysis, } \\
\text { chlorination, } \\
\text { photodegradation }\end{array}$ & $\begin{array}{l}16 \text { TPs of Cocaine ( } 3 \text { already } \\
\text { known), } 10 \text { TPs of BE ( } 1 \\
\text { already known) }\end{array}$ & - & - & UPLC-QqToF- MSE, HPLC-QqQ & $\begin{array}{l}\text { Waters MassLynx, MetaboLynx, XIC, } \\
\text { mass accuracy, fragmentation pattern }\end{array}$ & [72] \\
\hline Fenofibric acid & $\begin{array}{l}\text { Photodegradation: } \\
\mathrm{UV} \text { and } \mathrm{UV} / \mathrm{H}_{2} \mathrm{O}_{2}\end{array}$ & $\begin{array}{l}\text { 4-chloro-4'-(1-hydroxy-1- } \\
\text { methylethyl) benzophenone, \& } \\
\text { minor chlorinated aromatics } \\
\text { TPs }\end{array}$ & $\begin{array}{l}\text { algal growth } \\
\text { inhibition test } \\
\text { using } \\
\text { Pseudokirchneriella } \\
\text { subcapitata }\end{array}$ & - & LC-ESI-QTOF-MS/MS & $\begin{array}{l}\text { Agilent MassHunter Workstation, mass } \\
\text { accuracy, fragmentation pattern }\end{array}$ & [6] \\
\hline Imazalil & Ozonation & 4 TPs & $\begin{array}{l}\text { Acute toxicity test } \\
\text { Daphnia magna }\end{array}$ & - & LC- LTQ-Orbitrap-MS ${ }^{n}$ & $\begin{array}{l}\text { Thermo Xcalibur, XIC, mass accuracy, } \\
\text { fragmentation \& isotopic pattern, } \\
\text { retention time plausibility }\end{array}$ & [5] \\
\hline Ketoprofen & UV irradiation & 22 TPs & - & SPE (Oasis $\left.{ }^{\mathrm{TM}} \mathrm{HLB}\right)$ & $\begin{array}{l}\text { GC-MS, GC-Ion Trap-MS/MS, UPLC- } \\
\text { QqToF-MS }^{\mathrm{n}}\end{array}$ & $\begin{array}{l}\text { Agilent Chemstation, Varian MS } \\
\text { Workstation, Waters MassLynx, } \\
\text { MetaboLynx mass accuracy, } \\
\text { fragmentation pattern, elution order, } \\
\text { reference standards, NIST library }\end{array}$ & [73] \\
\hline Quinclorac & $\begin{array}{l}\text { Photolysis, } \\
\text { Photocatalysis } \\
\left(\mathrm{TiO}_{2}\right)\end{array}$ & $\begin{array}{l}14 \text { TPs: Pyridine ring } \\
\text { hydroxylation, ring opening } \\
\text { and/or oxidative } \\
\text { dechlorination }\end{array}$ & - & SPE (Oasis ${ }^{\mathrm{TM}}$ HLB) & HPLC-QTOF-MS/MS & $\begin{array}{l}\text { Agilent MassHunter, retention time } \\
\text { prediction: ChemBio3D Ultra, mass } \\
\text { accuracy, fragmentation \& isotopic } \\
\text { pattern }\end{array}$ & [74] \\
\hline $\begin{array}{l}8 \text { Triazines (ametryn, atrazine, } \\
\text { cyanazine, metrybuzine, } \\
\text { prometryn, propazin, } \\
\text { simazine, terbutryn) }\end{array}$ & $\begin{array}{l}\text { Chlorination with } \\
\text { sodium } \\
\text { hypochlorite }\end{array}$ & $\begin{array}{l}4 \text { TPs ( } 1 \text { never previously } \\
\text { reported) }\end{array}$ & $\begin{array}{l}\text { Acute toxicity test } \\
\text { Vibrio fischeri: TPs } \\
\text { higher toxicity }\end{array}$ & $\begin{array}{l}\text { On line SPE } \\
\text { (polymeric } \\
\text { cartridge) }\end{array}$ & HPLC-UV, UPLC-Q-ToF-MS/MS & $\begin{array}{l}\text { Waters MassLynx, mass accuracy, } \\
\text { fragmentation pattern }\end{array}$ & [75] \\
\hline
\end{tabular}




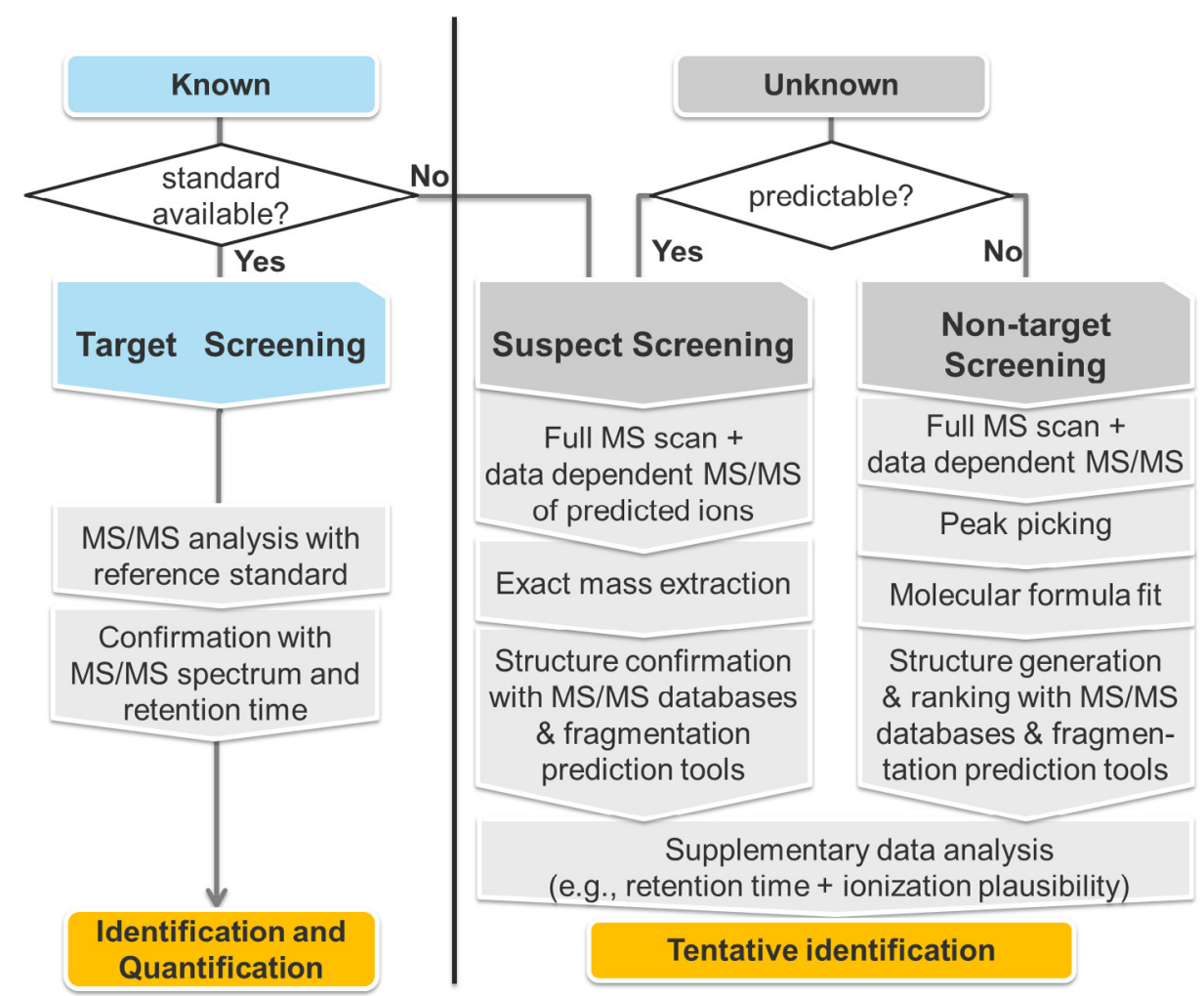

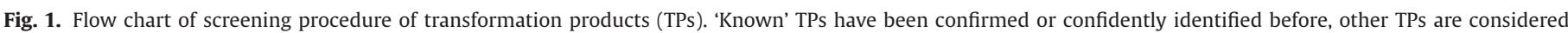
'Unknown'.

cartridge (weak anion, weak cation-exchange material, and nonpolar and hydrophilic-lipophilic interaction sorbents) [5].

As identification is a time-consuming process and often a great number of TPs are formed, prioritization of the most relevant TPs for identification and assessment is necessary. There are two main approaches:

- the effect-directed analysis (EDA); and,

- the exposure-driven approach.

The success of each approach underlies on the selection of the sample together with the sample preparation.

EDA, which has a toxicological perspective, was first introduced for the identification of TPs by Escher and Fenner in 2010 [8]. Modified EDA is based on the relation of the toxicity change of an environmental sample to the decrease of the parent-compound concentration, undergoing a transformation process. If increased toxicity is observed, then toxic TPs are likely to be formed [8].

Furthermore, the US EPA applies a standardized protocol for toxicity identification and evaluation (TIE) (www.epa.gov). EDA is a useful tool to identify non-target and unknown toxicants based on their effects on the environment. This approach works with stepwise separation and simplification of the sample in order to isolate components with toxic activity. The manipulations are directed by bioassays; when toxicity increases, identification of the toxic compound through chemical analysis should be performed. The fractionation approach is a non-selective, non-destructive, cleanup methodology.

However, the more common approach is exposure driven, where the TPs are distinguished and singled out for identification according to their occurrence. As the concentration of the TP gets higher, it is considered more relevant to risk. This approach is commonly used in the laboratory studies, followed by environmental fate assessment and toxicity testing [8].

The combination of both approaches is also possible:

- first, selection of samples with increased toxicity during transformation; and, subsequently,

- identification of the highest concentrated TPs in the sample without further separation.

\section{Identification approaches - analytical techniques}

Nowadays, liquid chromatography (LC) coupled to MS (LC-MS) using a variety of mass analyzers is the technique of choice for the investigation of EPs and TPs in environmental samples.

LC is a suitable chromatographic technique for polar, thermolabile compounds, thus for the identification of TPs, which are generally more polar than their parent molecules.

Mass analyzers commonly employed are triple quadrupole (QqQ), time-of-flight (TOF), ion-trap (IT), Orbitrap and hybrid [e.g., quadrupole time-of-flight (Q-TOF), quadrupole-linear ion trap (Q-LIT), linear ion trap-Orbitrap or quadrupole-Orbitrap].

There are various workflows in the literature for the identification of TPs, depending indispensably on the instrumentation and the available software. Fig. 1 shows the main outline:

(a) target analysis, which is based on the determination of already known TPs, and identification is carried out with standard solutions;

(b) suspect screening, with a list of possible TPs assembled from the literature or from prediction models, and the samples are screened for those candidates; and, 
(c) non-target screening, with identification of novel TPs being carried out with sophisticated post-acquisition data tools and supplementary analytical techniques.

The development and the use of powerful HR-MS is the driving force in development of novel analytical methodologies for the identification of TPs. Due to its sensitivity in full-scan acquisition mode and high mass accuracy, HR-MS is suitable for target and nontarget analysis, pre- and post-acquisition processing, retrospective analysis and discovery of TPs.

\subsection{Target analysis}

In target analysis, as shown in Fig. 1, TPs are already known and standards are available, so that they can be included within a defined MS method and be monitored in routine analysis. LC coupled to triple quadrupole (LC-QqQ-MS/MS) is the workhorse in target analysis. The QqQ analyzer permits application of MS/MS modes [e.g., production scan, precursor-ion scan, neutral-loss scan and selected reaction monitoring (SRM), which is the most predominant]. The SRM mode provides several advantages and interesting characteristics for target analysis, such as increased selectivity, reduced interferences and high sensitivity, which allows robust quantification. Another important point is the possibility of reducing the analysis time, including extraction and instrumental determination.

With the use of LC-QqQ-MS/MS, adequate results have been obtained for the analysis of ECs and the identification and the quantification of their TPs, especially in pesticides and pharmaceutical compounds, where standards are available.

HR-MS for target analysis offers promising solutions to the limitations of SRM analysis, which allows monitoring of only specific TPs. Virtually all compounds present in a sample can be determined simultaneously with HR-MS instruments operating in fullscan mode, making it unnecessary to pre-select compounds and associated SRM transitions. Target compounds included in a database are screened in the sample based on mass accuracy, isotopic pattern, retention time $\left(t_{R}\right)$ and MS/MS fragments. Alternatively, hybrid instruments offer the possibility of data-dependent MS/MS acquisition, where MS/MS analysis is triggered if a compound from a target-ion list is detected in the full scan. Moreover, HR-MS instruments can differentiate isobaric compounds with the same nominal mass but different molecular formula due to their higher resolving power $[3,12,13,15,16]$.

HR-MS outperforms LR-MS regarding the level of identification of an unknown compound, since, within Decision 2002/657/EC, it gains more identification points and can provide mass accuracy, even in full-scan mode. One ion in HR-MS gains two identification points, instead of one in LR-MS, whereas HR-MS ${ }^{\mathrm{n}}$ transition products gain two-and-a-half instead of one-and-a-half. It is clear that in HRMS full-scan mode, more than one ion is present in the mass spectra and evaluated.

\subsection{Suspect screening - prediction of transformation products}

Suspect screening is the technique of choice for the identification of TPs, when the confirmation of the analytes with a reference standard is impossible, but molecular formula and structure of suspected molecules can be predicted (Fig. 1) [10,13,15,50,51].

In suspect screening, an important step of the identification workflow is the prediction of possible TPs using computational (insilico) prediction tools. Commercially available or freely accessible programs have been applied in the prediction step on environmental analysis, including:

- University of Minnesota Pathway Prediction System (UM-PPS: http://eawag-bbd.ethz.ch/) [33,34];
- CATABOL (http://oasis-lmc.org/products/models/environmental -fate-and-ecotoxicity/catabol-301c.aspx);

- PathPred (http://www.genome.jp/tools/pathpred/); and,

- Meteor (http://www.lhasalimited.org/products/meteor -nexus.htm).

The prediction system should be properly selected by considering the organism or the system where TPs are formed.

Meteor was built based on mammalian biotransformation reactions of common functional groups and allows prediction of the most probable TPs, providing in parallel relevant literature references.

PathPred is a multi-step reaction prediction server for biodegradation pathways of xenobiotic compounds and biosynthesis pathways of secondary metabolites. It is linked to KEGG metabolic pathway maps and it has the potential to link the prediction result to genomic information.

CATABOL and UM-PPS predict microbial metabolic reactions based on biotransformation rules.

As UM-PPS is freely accessible and all rules applied are clearly assigned, it is the most common prediction tool in suspect screening, and many researchers have tried to evaluate and to improve its prediction power [34,50-52]. The prediction rules behind UMPPS come from the University of Minnesota Biocatalysis/ Biodegradation Database (UM-BBD) and literature [85]. Since UMBBD has integrated data generated from pure microbial cultures, the predicted pathways may not be completely appropriate for environmental systems [34]. The relatively high false-positive rates of all prediction systems are of concern, since the inclusion of additional pathways increases the number of possible degradation products. In UM-PPS, combinatorial explosion can be limited by prioritizing the different rules using relative reasoning.

Prediction of TPs is followed by the HR-MS analysis; the exact mass for each of the predicted TPs is extracted from the chromatogram and checked by comparing it with control samples. An intensity-threshold value is applied to cut off unclear spectra. The plausibility of the chromatographic $t_{R}$, isotopic pattern, and ionization efficiency are used as further filters to narrow down the number of candidate peaks. Furthermore, using MS/MS or $\mathrm{MS}^{\mathrm{n}}$, structures of suspected TPs are suggested based on the observed fragmentation pattern.

Depending on the above criteria, there are different confidence levels of identification in HR-MS analysis of TPs. When all the above criteria are fulfilled, a probable structure is proposed based on a library-spectrum match or diagnostic evidence. Otherwise, tentative candidates or just unequivocal molecular formulas are the outcome of suspect screening [86].

One approach for processing the data would be the identification of key TPs in terms of persistence over the time of the experiment. It is carried out by a data-processing method that is established based on peak detection, time-trend filtration and structure assignment. Open-source software is used for peak peaking (e.g., MZmine) and processing of the chromatograms (e.g., enviMass), by noise removal and blank subtraction. Then, a meaningful time trend is acquired and the remaining candidate peaks are compared with a list from UM-PPS or literature for tentative identification [87].

Another approach to suspect screening is based on the use of characteristic fragmentation undergone by EPs during MS/MS fragmentation events [51]. It is based on the assumption that many TPs maintain a structure similar to the parent compound and therefore have common fragment ions. Thus, searching for specific fragment ions in MS/MS spectra throughout the chromatographic run could lead to new TPs. This is evident when applying production and neutral-loss scans, and other techniques, such as massdefect filtering. 


\subsection{Non-target screening}

Non-target screening implies the identification of compounds for which there is no previous knowledge available and is usually carried out after target and suspect screening. Non-target screening becomes a challenging task, but, for TPs, further information of the parent compound (e.g., molecular formula, MS/MS spectrum, $t_{R}$ and other physico-chemical data) may contribute to further ranking of possible structures and facilitate the identification process [68]. For non-target screening, HR-MS is strongly required in order to have mass accuracy for confirmation of the molecular formula and reliable interpretation of the MS/MS spectra $[13,14]$.

The challenge with HR instruments is the generation of massive quantities of data and subsequently their evaluation and the export of results. Moreover, their ability to operate in fullscan and MS/MS modes simultaneously provides even more data in a single run. For this reason, post-acquisition data-processing tools are necessary; computer-aided techniques provide rapid, accurate and efficient data mining. There is a number of opensource and commercial software options for non-target screening, including:

- MZmine (http://mzmine.sourceforge.net/);

- XCMS (https://xcmsonline.scripps.edu);

- EnviMass (http://www.eawag.ch/forschung/uchem/software/ enviMass1);

- Non-target, ACD MS/Workbook Suite; and,

- vendors' software, such as Bruker Metabolite Tools and ProfileAnalysis, Waters MassLynx and MetaboLynx, Thermo Scientific Metworks and Sieve, Applied Biosystems Data Explorer (MDS-Sciex Analyst QS) and Agilent MassHunter.

The general procedure, as shown in Fig. 1, has several steps until it reaches the final result, which does not follow the same order in each software or workflow.

The first step is always peak picking. In this step, comparison of the sample with control or blank samples is important to exclude irrelevant peaks.

The removal of noise peaks, mass recalibration and componentization of isotopes and adducts is usually carried out automatically as the next step.

The assignment of the molecular formula to the accurate mass of the peak is performed using heuristic filters, such as the seven golden rules of Kind and Fiehn [88].

Exploration of databases, such as ChemSpider, PubChem, DAIOS database, NIST or structure generation, may lead to candidate structures $[89,90]$. Thereby, information on the parent compound (e.g., molecular formula, substructures) can help to restrict the search of databases and possible structures are likely to be proposed for the compound. However, databases contain mostly only EPs, but many TPs are not included yet.

One successful example of the application of a non-target workflow is the identification of biotransformation products of three benzotriazoles [36].

Even after filtering, strict criteria and thresholds in the above parameters, the number of peaks, which correspond to non-targets can exceed 1000. It is clear that elucidation of all those peaks would demand a great amount of time and effort; prioritization of the most intense peaks is a common strategy [91].

Similar to suspect screening, the observation of the presence or the absence of common characteristic ions in the fragmentation pattern of both the parent compound and the TPs, evidencing the stability or reactivity of certain parts of the molecule, can be helpful [3].

For ranking the candidate structures, information from MS/MS spectra has to be explored by comparing the fragmentation pattern with in-silico mass spectral fragmentation or with spectra in libraries. There are a few databases with mass spectra, e.g.:

- MassBank (http://massbank.ufz.de/MassBank/); and,

- MetLin (http://metlin.scripps.edu/index.php).

However, most software do not take into account the fragmentation pattern. MOLGEN-MS (http://www.molgen.de/), ACD/MS Fragmenter (www.acdlabs.com/products/adh/ms/ms_frag) and MassFrontier (www.highchem.com/index.php/massfrontier) use fragmentation rules, whereas MetFrag offers a purely combinatorial approach based on bond energies only. Although the overall candidate ranking with MetFrag is not quite as good as that obtained with Mass Frontier and MOLGEN-MS, the scoring function used in MetFrag can improve the ranking significantly (http:// msbi.ipb-halle.de/MetFrag/).

MetFusion, the newest development, combines MetFrag with spectral database searching (http://msbi.ipb-halle.de/MetFusion/).

The use of fragmentation trees as performed in SIRIUS is another approach for the structure elucidation (http://bio.informatik .uni-jena.de/sirius2/).

In any case, criteria must be established for the success of the identification of the TP by the accuracy of the molecular ion (e.g., mass error $<5 \mathrm{ppm}$, dependent on the mass accuracy) and the characteristic fragment ions in MS/MS mode (purity score $\geq 65$ recommended) [3].

Criteria also exist for the score of the isotopic pattern in most commercial software.

Müller et al. proposed another approach for non-target screening of TPs, focusing on relevant peaks (features). The sample is not regarded as an isolated specimen, but is rather evaluated in relation to a set of other samples based on considerations of, e.g., their temporal, spatial, or process-related connections. This also covers comparison of assays and controls, as carried out in evaluation of many transformation experiments. The features of the sample are considered as mathematical sets and treated with statistical tools [90].

Finally, orthogonal analytical approaches are frequently crucial for the successful unambiguous identification of TPs. Nuclear magnetic resonance (NMR) is one option for a complementary technique for identification and confirmation of TP structures as long as sufficient quantities can be isolated $[33,39]$.

\section{Future needs and trends}

Development of generic and retrospective analytical techniques should permit the simultaneous determination of parent compounds and their TPs, within a single run. In the identification of TPs, the future lies in tiered approaches, which employ HR-MS, complementary techniques and advanced software tools. HR-MS outperforms LR-MS, regarding the level of identification of an unknown compound. Moreover, identification by HR-MS analysis has different levels of confidence, regarding the supporting evidence recorded, apart from the molecular ion $\left\{\right.$ e.g., MS $^{\mathrm{n}}$ or library match [86]\}.

However, we are at an early stage in studying the identification of TPs and the workflows applied, as instrumentation and software are still in progress and are getting more complete and easier to use. Specific, step-wise, automated workflows that take into consideration the advantages of HR-MS instruments are still missing for suspect and non-target analysis. Exclusion parameters and sufficient filtering are necessary for the prioritization of peaks and elimination of false-positive and false-negative results. In a more integrated workflow, prediction models should also be combined and propose TPs from the whole range of transformation rules and automatically integrate them in subsequent suspect screening. 
Non-target screening should be an extra step for elucidating the rest of the unknown peaks, evaluating not only the most intense, but ideally the most relevant, also according to ecotoxicity. Therefore, new computational tools for toxicity prediction or coupling with high-throughput toxicity tests would be preferable.

Moreover, a universal $t_{R}$ prediction model, which does not need unreasonable efforts for calibration and takes into consideration neutral and ionic compounds, would contribute a lot in further evaluation of the data [32]. A gap also exists for in-silico fragmentation models, lacking mainly in giving fragments, reasonable for each ionization mode and according to specific rules.

However, definite identification can be performed only using reference standards, and is difficult because, in most cases, reference standards are not commercially available. Additional and supplementary orthogonal techniques (e.g., NMR, different MS techniques or other analytical or separation techniques) could contribute in the structure elucidation of unknown TPs, and in confirmation. In this way, multiple physicochemical properties of the compound would be taken into account [92]. Although, most non-target compounds reported are not fully identified, their communication in the scientific community is of great importance, especially if the results are accompanied by an uncertainty or a confidence level [92].

Despite the demands for an identification process, sample preparation should not be forgotten, since environmental samples are complicated matrices and TPs are expected at very low concentrations. It is clear that sample preparation is very challenging in order to capture the wide range of polarities of the potential TPs and to take full advantage of the analytical instruments but, at the same time, to eliminate interfering matrix components.

Summing up, researchers, scientists and policy makers still have a long way to go in order to explore the field of TPs of EPs and to perform an integrated risk assessment. Research is ongoing on the development of high-throughput techniques, including extraction, analysis and data-evaluation steps.

\section{Acknowledgments}

This project was implemented under the Greek Operational Program «Education and Lifelong Learning» and funded by the European Union (European Social Fund) and Greek National Resources (ARISTEIA 624).

\section{Appendix: Supplementary material}

Supplementary data to this article can be found online at doi:10.1016/j.trac.2014.11.009.

\section{References}

[1] C.G. Daughton, Non-regulated water contaminants: emerging research, Environ. Impact Assess. Rev. 24 (2004) 711-732.

[2] A.B.A. Boxall, The Handbook of Environmental Chemistry, vol. 2, P, Transformation Products of Synthetic Chemicals in the Environment, Springer, Berlin, 2009.

[3] A. Agüera, M. Jesús Martínez Bueno, A.R. Fernández-Alba, New trends in the analytical determination of emerging contaminants and their transformation products in environmental waters, Environ. Sci. Pollut. Res. 20 (2013) 34963515.

[4] M. la Farré, S. Pérez, L. Kantiani, D. Barceló, Fate and toxicity of emerging pollutants, their metabolites and transformation products in the aquatic environment, TRAC-Trend. Anal. Chem. 27 (2008) 991-1007.

[5] A.K. Genena, D.B. Luiz, W. Gebhardt, R.F.P.M. Moreira, H.J. José, H. Fr. Schröder, Imazalil degradation upon applying ozone-transformation products, kinetics, and toxicity of treated aqueous solutions, Ozone-Sci. Eng. 33 (2013) 308-328.

[6] J. Santiago, A. Agüera, M. del Mar Gómez-Ramos, A.R. Fernández Alba, E. García-Calvo, R. Rosal, Oxidation by-products and ecotoxicity assessment during the photodegradation of fenofibric acid in aqueous solution with UV and $\mathrm{UV} / \mathrm{H}_{2} \mathrm{O}_{2}$, J. Hazar. Mat. 194 (2011) 30-41.
[7] F.J. Benitez, J.L. Acero, F.J. Real, G. Roldan, E. Rodriguez, Photolysis of model emerging contaminants in ultra-pure water: kinetics, by-products formation and degradation pathways, Water Res. 47 (2013) 870-880.

[8] B.I. Escher, K. Fenner, Recent advances in environmental risk assessment of transformation products, Environ. Sci. Technol. 45 (2011) 3835-3847.

[9] F. Hernández, J.V. Sancho, M. Ibáñez, E. Abad, T. Portolés, L. Mattioli, Current use of high-resolution mass spectrometryin the environmental sciences, Anal. Bioanal. Chem. 403 (2012) 1251-1264.

[10] M. Krauss, H. Singer, J. Hollender, LC-high resolution MS in environmental analysis: from target screening to the identification of unknowns, Anal. Bioanal. Chem. 397 (2010) 943-951.

[11] M. Zedda, C. Zwiener, Is nontarget screening of emerging contaminants by LC-HRMS successful? A plea for compound libraries and computer tools, Anal. Bioanal. Chem. 403 (2012) 2493-2502.

[12] M.J. Gómez, M.M. Gómez-Ramos, O. Malato, M. Mezcua, A.R. Férnandez-Alba Rapid automated screening, identification and quantification of organic micro-contaminants and their main transformation products in wastewater and river waters using liquid chromatography-quadrupole-time-of-flight mass spectrometry with an accurate-mass database, J. Chromatogr. A 1217 (2010) 7038-7054.

[13] A.C. Hogenboom, J.A. van Leerdam, P. de Voogt, Accurate mass screening and identification of emerging contaminants in environmental samples by liquid chromatography-hybrid linear ion trap Orbitrap mass spectrometry, J. Chromatogr. A 1216 (2009) 510-519.

[14] M. Ibáñez, J.V. Sancho, O.J. Pozo, W. Niessen, F. Hernández, Use of quadrupole time-of-flight mass spectrometry in the elucidation of unknown compounds present in environmental water, Rapid Commun. Mass Spectrom. 19 (2005) 169-178.

[15] J. Nurmi, J. Pellinen, A.-L. Rantalainen, Critical evaluation of screening techniques for emerging environmental contaminants based on accurate mass measurements with time-of-flight mass spectrometry, J. Mass Spectrom. 47 (2012) 303-312.

[16] R. Díaz, M. Ibáñez, J.V. Sancho, F. Hernández, Target and non-target screening strategies for organic contaminants, residues and illicit substances in food, environmental and human biological samples by UHPLC-QTOF-MS, Anal. Methods 4 (2012) 196-209.

[17] S. Pérez, D. Barceló, Application of advanced MS techniques to analysis and identification of human and microbial metabolites of pharmaceuticals in the aquatic environment, TRAC-Trend. Anal. Chem. 26 (2007) 494514.

[18] M. Petrovic, D. Barceló, LC-MS for identifying photodegradation products of pharmaceuticals in the environment, TRAC-Trend. Anal. Chem. 26 (2007) 486-493.

[19] D. Fatta-Kassinos, M.I. Vasquez, K. Kümmerer, Transformation products of pharmaceuticals in surface waters and wastewater formed during photolysis and advanced oxidation processes - degradation, elucidation of byproducts and assessment of their biological potency, Chemosphere 85 (2011) 693709.

[20] B. de Witte, H. van Langenhove, K. Demeestere, J. Dewulf, Advanced oxidation of pharmaceuticals: chemical analysis and biological assessment of degradation products, Crit. Rev. Env. Sci. Tec. 41 (2011) 215-242.

[21] K. Levsen, H.-M. Schiebel, B. Behnke, R. Dötzer, W. Dreher, M. Elend, et al., Structure elucidation of phase II metabolites by tandem mass spectrometry: an overview, J. Chromatogr. A 1067 (2005) 55-72.

[22] F. Hernández, J.V. Sancho, M. Ibáñez, S. Grimalt, Investigation of pesticide metabolites in food and water by LC-TOF-MS, Trends Anal. Chem. 27 (2008) 862-872.

[23] J.L. Martínez Vidal, P. Plaza-Bolaños, R. Romero-González, A. Garrido Frenich, Determination of pesticide transformation products: a review of extraction and detection methods, J. Chromatogr. A 1216 (2009) 6767-6788.

[24] J.M. Horvat, M. Petrović, S. Babić, D.M. Pavlović, D. Ašperger, S. Pelko, et al. Analysis, occurrence and fate of anthelmintics and their transformation products in the environment, TRAC-Trend. Anal. Chem. 31 (2012) 61-84.

[25] J.M. Santos, M.S. Miranda, J.C.G. Esteves da Silva, The degradation products of UV filters in aqueous and chlorinated aqueous solutions, Water Res. 46 (2012) 3167-3176.

[26] P. Gago-Ferrero, M.S. Díaz-Cruz, D. Barceló, Liquid chromatography-tandem mass spectrometry for the multi-residue analysis of organic UV filters and their transformation products in the aquatic environment, Anal. Methods 5 (2013) 355-366.

[27] H.N. Bhatti, R.A. Khera, Biological transformations of steroidal compounds: a review, Steroids 77 (2012) 1267-1290.

[28] H. Hühnerfuss, M. Raza Shah, Enantioselective chromatography-A powerful tool for the discrimination of biotic and abiotic transformation processes of chiral environmental pollutants, J. Chromatogr. A 1216 (2009) 481-502.

[29] M. Farré, L. Kantiani, M. Petrovic, S. Pérez, D. Barceló, Achievements and future trends in the analysis of emerging organic contaminants in environmental samples by mass spectrometry and bioanalytical techniques, J. Chromatogr. A 1259 (2012) 86-99.

[30] K. Fischer, E. Fries, W. Körner, C. Schmalz, C. Zwiener, New developments in the trace analysis of organic water pollutants, Appl. Microbiol. Biotechnol. 94 (2012) 11-28

[31] C. Moschet, A. Piazzoli, H. Singer, J. Hollender, Alleviating the reference standard dilemma using a systematic exact mass suspect screening approach with liquid chromatography-high resolution mass spectrometry, Anal. Chem. 85 (2013) $10312-10320$ 
[32] C. Hug, N. Ulrich, T. Schulze, W. Brack, M. Krauss, Identification of novel micropollutants in wastewater by a combination of suspect and nontarget screening, Environ. Pollut. 184 (2014) 25-32.

[33] C. Prasse, M. Wagner, R. Schulz, T.A. Ternes, Biotransformation of the antiviral drugs acyclovir and penciclovir in activated sludge treatment, Environ. Sci. Technol. 45 (2011) 2761-2769.

[34] D. Helbling, J. Hollender, H.-P. Kohler, K. Fenner, Structure-based interpretation of biotransformation pathways of amide-containing compounds in sludgeseeded bioreactors, Environ. Sci. Technol. 44 (2010) 6628-6635.

[35] A. Pérez-Parada, A. Agüera, M. del Mar Gómez-Ramos, J.F. García-Reyes, H. Heinzen, A.R. Fernández-Alba, Behavior of amoxicillin in wastewater and river water: identification of its main transformation products by liquid chromatography/electrospray quadrupole time-of-flight mass spectrometry, Rapid Commun. Mass Spectrom. 25 (2011) 731-742.

[36] S. Huntscha, T.B. Hofstetter, E.L. Schymanski, S. Spahr, J. Hollender, Biotransformation of benzotriazoles: insights from transformation product identification and compound-specific isotope analysis, Environ. Sci. Technol. 48 (2014) 4435-4443.

[37] A. Jelic, C. Cruz-Morato, E. Marco-Urrea, M. Sarra, S. Perez, T. Vicent, et al., Degradation of carbamazepine by Trametes versicolor in an air pulsed fluidized bed bioreactor and identification of intermediates, Water Res. 46 (2012) 955-964.

[38] C. Trautwein, K. Kümmerer, Degradation of the tricyclic antipsychotic drug chlorpromazine under environmental conditions, identification of its main aquatic biotic and abiotic transformation products by LC-MS ${ }^{\mathrm{n}}$ and their effects on environmental bacteria, J. Chromatogr. B 889-890 (2012) 24-38.

[39] A. Wick, M. Wagner, T.A. Ternes, Elucidation of the transformation pathway of the opium alkaloid codeine in biological wastewater treatment, Environ. Sci. Technol. 45 (2011) 3374-3385.

[40] E. Marco-Urrea, M. Pérez-Trujillo, C. Cruz-Morató, G. Caminal, T. Vicent, Degradation of the drug sodium diclofenac by Trametes versicolor pellets and identification of some intermediates by NMR, J. Hazar. Mat. 176 (2010) 836-842.

[41] S. Pérez, D. Barceló, First evidence for occurrence of hydroxylated human metabolites of diclofenac and aceclofenac in wastewater using QqLIT-MS and QqTOF-MS, Anal. Chem. 80 (2008) 8135-8145.

[42] M. Shen, J. Cheng, R. Wu, S. Zhang, L. Mao, S. Gao, Metabolism of polybrominated diphenyl ethers and tetrabromobisphenol A by fish liver subcellular fractions in vitro, Aquat. Toxicol. 114-115 (2012) 73-79.

[43] L. Zhao, P.W. Folsom, B.W. Wolstenholme, H. Sun, N. Wang, R.C. Buck, 6:2 Fluorotelomer alcohol biotransformation in an aerobic river sediment system, Chemosphere 90 (2013) 203-209.

[44] N. Wang, R.C. Buck, B. Szostek, L.M. Sulecki, B.W. Wolstenholme, 5:3 Polyfluorinated acid aerobic biotransformation in activated sludge via novel "one-carbon removal pathways, Chemosphere 87 (2012) 527-534.

[45] S. Terzic, I. Senta, M. Matosic, M. Ahel, Identification of biotransformation products of macrolide and fluoroquinolone antimicrobials in membrane bioreactor treatment by ultrahigh-performance liquid chromatography/ quadrupole time-of-flight mass spectrometry, Anal. Bioanal. Chem. 401 (2011) 353-363.

[46] J.L. Kormos, M. Schulz, M. Wagner, T.A. Ternes, Multistep approach for the structural identification of biotransformation products of iodinated X-ray contrast media by liquid chromatography/hybrid triple quadrupole linear ion trap mass spectrometry and ${ }^{1} \mathrm{H}$ and ${ }^{13} \mathrm{C}$ nuclear magnetic resonance, Anal. Chem. 81 (2009) 9216-9224.

[47] J.L. Kormos, M. Schulz, H.-P. Kohler, T.A. Ternes, Biotransformation of selected iodinated X-ray contrast media and characterization of microbial transformation pathways, Environ. Sci. Technol. 44 (2010) 4998-5007.

[48] M. Badia-Fabregat, C.E. Rodríguez-Rodríguez, P. Gago-Ferrero, A. Olivare, B. Piña, M.S. Díaz-Cruz, et al., Degradation of UV filters in sewage sludge and 4-MBC in liquid medium by the ligninolytic fungus Trametes versicolor, J. Environ. Manage. 104 (2012) 114-120.

[49] C. Trautwein, K. Kümmerer, Incomplete aerobic degradation of the antidiabetic drug Metformin and identification of the bacterial dead-end transformation product Guanylurea, Chemosphere 85 (2011) 765-773.

[50] D. Helbling, J. Hollender, H.P. Kohler, H. Singer, K. Fenner, High-throughput identification of microbial transformation products of organic micropollutants, Environ. Sci. Technol. 44 (2010) 6621-6627.

[51] S. Kern, K. Fenner, H.P. Singer, R.P. Schwarzenbach, J. Hollender, Identification of transformation products of organic contaminants in natural waters by computer-aided prediction and high-resolution mass spectrometry, Environ. Sci. Technol. 43 (2009) 7039-7046.

[52] S. Kern, R. Baumgartner, D.E. Helbling, J. Hollender, H. Singer, M.J. Loos, et al., A tiered procedure for assessing the formation of biotransformation products of pharmaceuticals and biocides during activated sludge treatment, J. Environ. Monit. 12 (2010) 2100-2111.

[53] J.B. Quintana, S. Weiss, T. Reemtsma, Pathways and metabolites of microbial degradation of selected acidic pharmaceutical and their occurrence in municipal wastewater treated by a membrane bioreactor, Water Res. 39 (2005) 2654-2664.

[54] C.E. Rodríguez-Rodríguez, M.J. García-Galán, P. Blánquez, M.S. Díaz-Cruz, D. Barceló, G. Caminal, et al., Continuous degradation of a mixture of sulfonamides by Trametes versicolor and identification of metabolites from sulfapyridine and sulfathiazole, J. Hazar. Mat. 213-214 (2012) 347-354.

[55] M. Fahrbach, M. Krauss, A. Preiss, H.P.E. Kohler, J. Hollender, Anaerobic testosterone degradation in Steroidobacter denitrificans - identification of transformation products, Environ. Pollut. 158 (2010) 2572-2581.
[56] K. Murugesan, Y.Y. Chang, Y.-M. Kim, J.R. Jeon, E.J. Kim, Y.S. Chang, Enhanced transformation of triclosan by laccase in the presence of redox mediators, Water Res. 44 (2010) 298-308.

[57] Y.M. Kim, K. Murugesan, S. Schmidt, V. Bokare, J.R. Jeon, E.J. Kim, et al., Triclosan susceptibility and co-metabolism - a comparison for three aerobic pollutantdegrading bacteria, Bioresour. Technol. 102 (2011) 2206-2212.

[58] D. Helbling, D. Johnson, M. Honti, K. Fenner, Micropollutant biotransformation kinetics associate with WWTP process parameters and microbial community characteristics, Environ. Sci. Technol. 46 (2012) 10579-10588.

[59] J.S.C. Liou, B. Szostek, C.M. DeRito, E.L. Madsen, Investigating the biodegradability of perfluorooctanoic acid, Chemosphere 80 (2010) 176-183.

[60] S. Vaalgamaa, A.V. Vahatalo, N. Perkola, S. Huhtala, Photochemical reactivity of perfluorooctanoic acid (PFOA) in conditions representing surface water, Sci. Total Environ. 409 (2011) 3043-3048.

[61] S. Reddy, C.R. Iden, B.J. Brownawell, Analysis of steroid conjugates in sewage influent and effluent by liquid chromatography-tandem mass spectrometry, Anal. Chem. 77 (2005) 7032-7038.

[62] V. Kumar, A.C. Johnson, N. Nakada, N. Yamashita, H. Tanaka, De-conjugation behavior of conjugated estrogens in the raw sewage, activated sludge and river water, J. Hazar. Mat. 227 (2012) 49-54.

[63] L. Lishman, S.A. Smyth, K. Sarafin, S. Kleywegt, J. Toito, T. Peart, et al., Occurrence and reductions of pharmaceuticals and personal care products and estrogens by municipal wastewater treatment plants in Ontario, Canada, Sci. Total Environ. 367 (2006) 544-558.

[64] E.M. Thurman, I. Ferrer, Liquid chromatography/quadrupole-time-of-flight mass spectrometry with metabolic profiling of human urine as a tool for environmental analysis of Dextromethorphan, J. Chromatogr. A 1259 (2012) $158-166$.

[65] I. Ferrer, E.M. Thurman, Identification of a new antidepressant and its glucuronide metabolite in water samples using liquid chromatography/ quadrupole time-of-flight mass spectrometry, Anal. Chem. 82 (2010) 81618168.

[66] E.M. Smith, S. Chu, G. Paterson, C.D. Metcalfe, J.Y. Wilson, Cross-species comparison of fluoxetine metabolism with fish liver microsomes, Chemosphere 79 (2010) 26-32.

[67] L.L. Johnson, B.F. Anulacion, M.R. Arkoosh, D.G. Burrows, D.A.M. Da silva, J.P. Dietrich, et al., Effects of legacy persistent organic pollutants (POPs) in fish current and future challenges, in: K. Tierney, A. Farrell, C. Brauner (Editors), Organic Chemical Toxicology of Fishes, vol. 33, Fish Physiology, Elsevier Inc, 2014, pp. 53-140.

[68] J. Jeon, D. Kurth, J. Hollender, Biotransformation pathways of biocides and pharmaceuticals in freshwater crustaceans based on structure elucidation of metabolites using high resolution mass spectrometry, Chem. Res. Toxicol. 26 (2013) 313-324.

[69] R. Ashauer, A. Hintermeister, I. O’Connor, M. Elumelu, J. Hollender, B.I. Escher, Significance of xenobiotic metabolism for bioaccumulation kinetics of organic chemicals in Gammarus pulex, Environ. Sci. Technol. 46 (2012) 3498-3508.

[70] L. Tong, P. Eichhorn, S. Purez, Y. Wanga, D. Barceló, Photodegradation of azithromycin in various aqueous systems under simulated and natural solar radiation: kinetics and identification of photoproducts, Chemosphere 83 (2011) 340-348.

[71] A. Müller, S.C. Weiss, J. Beißwenger, H.G. Leukhardt, W. Schulz, W. Seitz, et al., Identification of ozonation by-products of 4- and 5-methyl- 1H-benzotriazole during the treatment of surface water to drinking water, Water Res. 46 (2012) 679-690.

[72] L. Bijlsma, C. Boix, W.M.A. Niessen, M. Ibáñez, J.V. Sancho, F. Hernández, Investigation of degradation products of cocaine and benzoylecgonine in the aquatic environment, Sci. Total Environ. 443 (2013) 200-208.

[73] T. Kosjek, S. Perko, E. Heath, B. Kralj, D. Zigon, Application of complementary mass spectrometric techniques to the identification of ketoprofen phototransformation products, J. Mass Spectrom. 46 (2011) 391-401.

[74] L. Pareja, A. Pérez-Parada, A. Agüera, V. Cesio, H. Heinzen, A.R. Fernández-Alba, Photolytic and photocatalytic degradation of quinclorac in ultrapure and paddy field water: identification of transformation products and pathways, Chemosphere 87 (2012) 838-844.

[75] R. Brix, N. Bahi, M.J. Lopez de Alda, M. Farré, J.-M. Fernandez, D. Barceló, Identification of disinfection by-products of selected triazines in drinking water by LC-Q-ToF-MS/MS and evaluation of their toxicity, J. Mass Spectrom. 44 (2009) 330-337.

[76] V. Homem, L. Santos, Degradation and removal methods of antibiotics from aqueous matrices - a review, J. Environ. Manage. 92 (2011) 2304-2347.

[77] R. Oliveira Pereira, C. Postigo, M. López de Alda, L.A. Daniel, D. Barceló, Removal of estrogens through water disinfection processes and formation of by-products, Chemosphere 82 (2011) 789-799.

[78] S.G. Zimmermann, M. Wittenwiler, J. Hollender, M. Krauss, C. Ort, H. Siegrist, et al., Kinetic assessment and modeling of an ozonation step for full-scale municipal wastewater treatment: micropollutant oxidation, by-product formation and disinfection, Water Res. 45 (2011) 605-617.

[79] N. Hamdi El Najjar, M. Deborde, R. Journel, N. Karpel Vel Leitner, Aqueous chlorination of levofloxacin: kinetic and mechanistic study, transformation product identification and toxicity, Water Res. 47 (2013) 121-129.

[80] C. Sirtori, A. Zapata, S. Malato, A. Agüera, Formation of chlorinated by-products during photo-Fenton degradation of pyrimethanil under saline conditions. Influence on toxicity and biodegradability, J. Hazard. Mater. 217-218 (2012) 217-223. 
[81] D. Sud, P. Kaur, Heterogeneous photocatalytic degradation of selected organophosphate pesticides: a review, Crit. Rev. Env. Sci. Tec. 42 (2012) 2365-2407.

[82] O.K. Dalrymple, D.H. Yeh, M.A. Trotz, Removing pharmaceuticals and endocrinedisrupting compounds from wastewater by photocatalysis, J. Chem. Technol. Biotechnol. 82 (2007) 121-134.

[83] A.J.M. Santos, M.S. Miranda, J.C.G. Esteves da Silva, The degradation products of UV filters in aqueous and chlorinated aqueous solutions, Water Res. 46 (2012) 3167-3176.

[84] E. Grabowska, J. Reszczyńska, A. Zaleska, Mechanism of phenol photodegradation in the presence of pure and modified- $\mathrm{TiO}_{2}$ : a review, Water Res. 46 (2012) 5453-5471.

[85] L.B.M. Ellis, J. Gao, K. Fenner, L.P. Wackett, The University of Minnesota pathway prediction system: predicting metabolic logic, Nucleic Acids Res. 36 (2008) W427-W432.

[86] E.L. Schymanski, J. Jeon, R. Gulde, K. Fenner, M. Ruff, H.P. Singer, et al., Identifying small molecules via high resolution mass spectrometry: communicating confidence, Environ. Sci. Technol. 48 (2014) 2097-2098.

[87] Z. Li, M.P. Maier, M. Radke, Screening for pharmaceutical transformation products formed in river sediment by combining ultrahigh performance liquid chromatography/high resolution mass spectrometry with a rapid dataprocessing method, Anal. Chim. Acta 810 (2013) 61-70.

[88] T. Kind, O. Fiehn, Seven Golden Rules for heuristic filtering of molecular formulas obtained by accurate mass spectrometry, BMC Bioinformatics 8 (2007) 105-106.

[89] J.L. Little, A.J. Williams, A. Pshenichnov, V. Tkachenko, Identification of "known unknowns" utilizing accurate mass data and ChemSpider, J. Am. Soc. Mass Spectrom. 23 (2012) 179-185.

[90] A. Müller, W. Schulz, W.K.L. Ruck, W.H. Weber, A new approach to data evaluation in the non-target screening of organic trace substances in water analysis, Chemosphere 85 (2011) 1211-1219.

[91] E.L. Schymanski, H.P. Singer, P. Longrée, M. Loos, M. Ruff, M.A. Stravs, et al., Strategies to characterize polar organic contamination in wastewater: exploring the capability of high resolution mass spectrometry, Environ. Sci. Technol. 48 (2014) 1811-1818.

[92] D.J. Creek, W.B. Dunn, O. Fiehn, J.L. Griffin, R.D. Hall, Z. Lei, et al., Metabolite identification: are you sure? And how do your peers gauge your confidence?, Metabolomics 10 (2014) 350-353. 